\title{
Better Contrast for Imaging Defects by ABF
}

\author{
Peng Gao ${ }^{1,2,3 *}$, Ryo Ishikawa ${ }^{4}$, Bin Feng $^{4}$, Naoya Shibata ${ }^{4}$, Yuichi Ikuhara ${ }^{4,5,6 *}$ \\ ${ }^{1}$ Electron microscopy laboratory, School of Physics, and Center for Nanochemistry, Peking University, \\ Beijing 100871, China \\ ${ }^{2}$ Collaborative Innovation Center of Quantum Matter, Beijing 100871, China \\ ${ }^{3}$ Center for Nanochemistry, Peking University, Beijing 100871, China \\ ${ }^{4}$ Institute of Engineering Innovation, The University of Tokyo, Tokyo 113-8656, Japan \\ ${ }^{5}$ Nanostructures Research Laboratory, Japan Fine Ceramic Center, Nagoya 456-8587, Japan \\ ${ }^{6}$ WPI Advanced Institute for Materials Research, Tohoku University, Sendai 980-8577, Japan
}

Structural defects in solid materials play very important roles in determing the physical and chemical properties. In particular, in the devices with nanometer scale, single defect can dominate the entire response. The properties of the defects are controlled by the microstructures. Therefore, there are great research interests to reveal the structure of defects. The transmission electron microscope is one of most important tools for defect characterization. In particular, the recent advancements of the annular bright field (ABF) imaging [1,2] in an aberration corrected scanning transmission electron microscope allow us to simultaneously determine both the heavier and lighter atom positions in oxides over a wide range of thickness, providing unprecedented opportunities to advance our understanding of the structure and properties of defects.

Here, we use ABF to study the diverse type of defects in oxides. Surface is one of the most important two-dimensional defects. At the surface of ferroelectrics, due to the strong coupling between the lattice and charge, the broken continuities of polarization vectors can cause local structure relaxation and charge redistribution to screen the polarization charge, which can significantly alter the properties. The commonly used surface probe technique is scanning tunneling microscopy (STM), which is based on the tunneling current and requires good conductivity, is no longer suitable to study these insulating ferroelectrics. By using the atomically resolved ABF imaging, we quantitatively measure the local polarization at the surface in $\mathrm{Pb}\left(\mathrm{Zr}_{0.2} \mathrm{Ti}_{0.8}\right) \mathrm{O}_{3}$ thin film [3]. We find that the structure of surface layer is governed by the polarization underneath. The thickness and atomic structure of surface layer between the negatively poled surface, positively poled surface, and neutral surface are completely different. Formation of these complicated surface configurations in different domains is driven by the screen mechanism at the surfaces.

The dislocation and grain boundary cores in $\mathrm{SrTiO}_{3}$ bicrystals are also studied by using ABF imaging. The atomic structure of dislocation cores including the oxygen positions is determined. There are two different types of dislocation cores, i.e., the $\mathrm{SrO}$ plane terminated and $\mathrm{TiO}_{2}$ plane terminated. Although they have the same Burgers vector of a[100], the atomic arrangements are different. Similar, the atomic arrangement of the grain boundary including oxygen is also identified.

Compared to the high angle annular dark field (HAADF) image, besides the ability to simultaneously image both heave and light atoms, the ABF also has much better contrast for imaging the atomic columns at the defects. At the defects, low occupancy, misalignments (induced by structure distortion), and/or large interdistance usually occur, leading to much weaker channeling effect. The HAADF image 
usually gives poor contrast for defects because the thermal diffuse scattered (TDS) electrons strongly depend on the atomic number (Z-contrast, $\mathrm{Z}$ is atomic number). Unlike HAADF, the signal in the ABF comes from both the TDS and elastic scattering [4], making ABF contrast is less dependent on Z. But still, the ABF contrast is robust enough to ensure no contrast reversal with changing the thickness. Therefore, the ABF imaging provides better contrast to image these defects.

In summary, there are at least two advantages for ABF to image the defects in oxides. First, both the heavier cations and lighter oxygen positions can be determined simultaneously. Second, the ABF gives much better contrast for the atomic columns in the dislocation and grain boundary cores, and at the surface.

\section{References:}

[1] E. Okunishi et al, Microsc.Microanal. 15 (Suppl 2) (2009) 164.

[2] S. Findlay et al, App.Phys.Lett., 95 (2009) 191913.

[3] P. Gao et al, Nature Communications, 7 (2016) 11318.

[4]The authors gratefully acknowledge the financial support through a Grant-in-Aid for Scientific Research on Innovative Areas "Nano Informatics" (Grant No. 25106003) from Japan Society for the Promotion of Science (JSPS), and "Nanotechnology Platform" (Project No. 12024046) from the Ministry of Education, Culture, Sports, Science and Technology in Japan (MEXT), the National Natural Science Foundation of China (51672007, 51502007), National Basic Research Program of China (2016YFA0300804, 2016YFA0300903), and "2011 Program" Peking-Tsinghua-IOP Collaborative Innovation Centre for Quantum Matter.

a

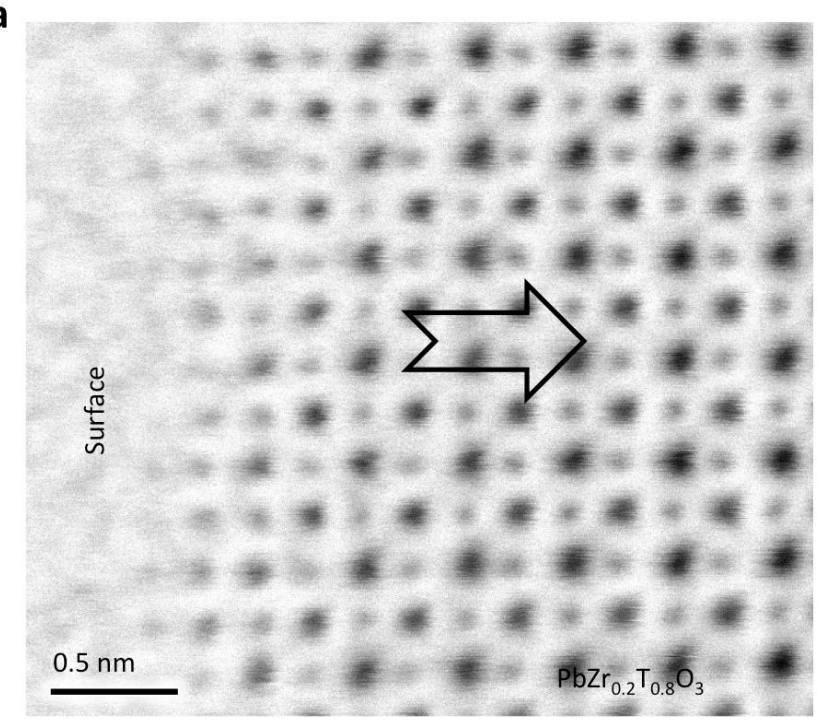

b

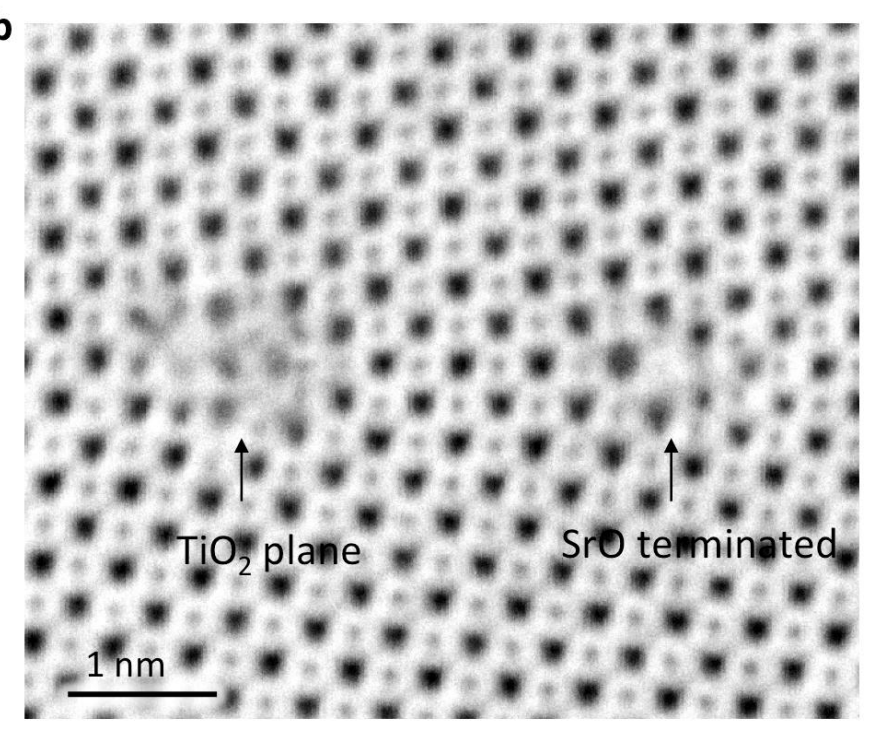

Figure 1. (a) Atomically resolved ABF image of $\mathrm{PbZr}_{0.2} \mathrm{Ti}_{0.8} \mathrm{O}_{3}$ thin film on $\mathrm{SrTiO}_{3}$ substrate. (b) Calculated $\mathrm{Pb}-\mathrm{O}$ bond length for the negatively poled surface. (c) Calculated $\mathrm{Pb}-\mathrm{O}$ bond length for the positively poled surface. (d) Calculated $\mathrm{Pb}-\mathrm{O}$ bond length for the neutral surface. 